\title{
A convergence result on random products of mappings in metric spaces
}

\author{
Mohamed Amine Khamsi ${ }^{1,2^{*}}$ and Issam Louhichi ${ }^{2}$
}

\author{
* Correspondence: \\ mohamed@utep.edu \\ 'Department of Mathematical \\ Sciences, The University of Texas At \\ El Paso, El Paso, TX 79968, USA \\ Full list of author information is \\ available at the end of the article
}

\begin{abstract}
Let $X$ be a metric space and $\left\{T_{1}, \ldots, T_{N}\right\}$ be a finite family of mappings defined on $D \subset$ $X$. Let $r: \mathbb{N} \rightarrow\{1, \ldots, N\}$ be a map that assumes every value infinitely often. The purpose of this article is to establish the convergence of the sequence $\left(x_{n}\right)$ defined by

$$
x_{0} \in D ; \text { and } x_{n+1}=T_{r(n)}\left(x_{n}\right), \text { for all } n \geq 0 \text {. }
$$

In particular, we extend the study of Bauschke [1] from the linear case of Hilbert spaces to metric spaces. Similarly we show that the examples of convergence hold in the absence of compactness. These type of methods have been used in areas like computerized tomography and signal processing.

Mathematical Subject Classification (2000): Primary 47H09; Secondary 46C99, 47N10, 65F10, 65K05, 90C25, 92C55.

Keywords: computerized tomography, convex feasibility problem, convex programming, Fejér monotone sequence, image reconstruction, image recovery, innate bounded regularity, Kaczmarz's method, nonexpansive mapping, projection algorithm, projective mapping, random product, signal processing, unrestricted iteration, unrestricted product
\end{abstract}

\section{Introduction}

Many problems in mathematics [2] and physical sciences [3-5] uses a technique known as finding common fixed point. Let $X$ be a real Hilbert space and suppose $T_{1}, \ldots, T_{N}$ are pairwise distinct self-mappings of some nonempty and closed subset $D$ of $X$. Suppose further that the fixed point set, $\operatorname{Fix}\left(T_{i}\right)=\left\{x \in D: T_{i}(x)=x\right\}$, of each mapping $T_{i}$ is nonempty and that $C=\operatorname{Fix}\left(T_{1}\right) \cap \cdots \cap \operatorname{Fix}\left(T_{N}\right) \neq \emptyset$. The aim is to find a common fixed point of these mappings. One frequently employed approach is the following:

Let $r$ be a random mapping for $\{1, \ldots ., N\}$, i.e., a surjective mapping from $\mathbb{N}$ onto $\{1, \ldots$, $N\}$ that takes each value in $\{1, \ldots, N\}$ infinitely often. Then generate a random sequence $\left(x_{n}\right)_{n}$ by taken $x_{0} \in D$ arbitrary, and

$$
x_{n+1}=T_{r(n)}\left(x_{n}\right), \text { for all } n>0,
$$

and hope that this sequence converges to a point in $C$. We call it a random or unrestricted product (resp. iteration). For products generated by using control 
sequence, there are many results: for instance, cyclic control arises when $r(n)=n+1$ $\bmod N$ (see, for example, [6]).

In general, this random product fails to have well convergence behavior. The first positive results were done in the case, when $D=X$ and each mapping $T_{i}$, is the projection onto nonempty, closed, and convex subset $C_{i}$ of $X$; hence $\operatorname{Fix}\left(T_{i}\right)=C_{i}, i=1, \ldots, N$. The problem of finding a common fixed point is then the well-known Convex Feasibility Problem (see, for example, [6]). In [7], there are several interesting applications of this problem. Some of the early known results in this case are:

1. Amemiya and Ando [8]: If each set $C_{i}$, is a closed subspace, then the random product converges weakly to the projection onto $C$.

2. Bruck [9]: If some set $C_{i}$, is compact, then the random product converges in norm to a point in $C$. If $N=3$ and each set $C_{i}$, is symmetric, then the random product converges weakly to some point in $C$.

3. Dye [10]: If the sets $C_{i}$, are finite-dimensional subspaces, then the random product converges in norm to a point in $C$.

4. Dye and Reich [11]: If the sets $C_{i}$, have a common weak internal point or if $N=$

3 , then the random product converges weakly to a point in $C$.

5. Youla [12]: If the sets $C_{i}$, have a common "inner point", then the random product converges weakly to a point in $C$.

6. Aharoni and Censor [13], Flam and Zowe [14], Tseng [15], and Eisner et al. [16]: If $X$ is finite dimensional, then the random product converges in norm to a point in $C$.

For more recent references, one can consult [17] and the references therein.

Dye et al. [18] were successful in their extension of Amemiya and Ando's results from Hilbert spaces to Banach spaces. In this study, we try to do the same by extending Bauschke's results [1] from linear Hilbert spaces to nonlinear metric spaces.

\section{Basic definitions and results}

Definition 2.1. Let $(X, d)$ be a metric space and $D$ be a nonempty subset of $X$. The mapping $T: D \rightarrow D$ is said to be projective w.r.t. $c_{0} \in \operatorname{Fix}(T)$ whenever

(i) $d(T(x), c) \leq d(x, c)$, for any $x \in D$, and any $c \in \operatorname{Fix}(T)$;

(ii) for any bounded sequence $\left(x_{n}\right)_{n} \subset D$, we have

$$
\lim _{n \rightarrow \infty}\left[d\left(x_{n}, c_{0}\right)-d\left(T\left(x_{n}\right), c_{0}\right)\right]=0 \Rightarrow \lim _{n \rightarrow \infty} d\left(x_{n}, \operatorname{Fix}(T)\right)=0 .
$$

Moreover $T$ is called a projective mapping if and only if $T$ is projective w.r.t. any point in $\operatorname{Fix}(T)$.

It is worth to mention that in [1] it is assumed that $T$ is nonexpansive to be projective. In particular it is not immediate that $T$ is continuous under our definition.

Example 2.1. Let $X$ be a Hilbert space and $Y$ be a closed subspace of $X$. Let $P: X \rightarrow$ $Y$ be the orthogonal projection. Then $\operatorname{Fix}(P)=Y$. For any $x \in X$ and $c \in Y$, we have

$$
\|x-c\|^{2}=\|P(x)-c\|^{2}+\|x-P(x)\|^{2} .
$$


Let $\left(x_{n}\right)_{n}$ be a bounded sequence such that $\lim _{n \rightarrow \infty}\left[\left\|x_{n}-c\right\|-\left\|P\left(x_{n}\right)-c\right\|\right]=0$, for some $c \in Y$. Then we have

$$
\lim _{n \rightarrow \infty}\left\|x_{n}-P\left(x_{n}\right)\right\|=0 .
$$

Since $P(x) \in \operatorname{Fix}(P)$, for any $x \in X$, we conclude that $\lim _{n \rightarrow \infty} d\left(x_{n}, \operatorname{Fix}(P)\right)=0$. In other words, $P$ is a projective mapping. This example explains why the word projective is used in the above definition.

More examples of projective mappings may be found in [1]. We have the following result about projective mappings.

Proposition 2.1. Let $(X, d)$ be a complete metric space and $D$ be a nonempty and closed subset of $X$. Let $T: D \rightarrow D$ be a projective mapping. Then for any $x \in D$, the iterates $\left(T^{n}(x)\right)_{n}$ converge. Set $P(x)=\lim _{n \rightarrow \infty} T^{n}(x)$. Then $P: D \rightarrow \operatorname{Fix}(T)$ is a retract which satisfies

(i) $P \circ T=T \circ P=P$;

(ii) $d(P(x), c) \leq d(x, c)$, for any $x \in D$, and $c \in \operatorname{Fix}(T)$.

Moreover $P$ is nonexpansive whenever $T$ is nonexpansive.

Proof. Let $x \in D$ and consider the iterates $\left(T^{n}(x)\right)_{n}$. Then from the assumptions on $T$, we can see that $\left(d\left(T^{n}(x), c\right)\right)_{n}$ is decreasing. So this sequence must be convergent. In particular we must have $\lim _{n \rightarrow \infty}\left[d\left(T^{n}(x), c\right)-d\left(T^{n+1}(x), c\right)\right]=0$. Hence $\lim _{n \rightarrow \infty} d\left(T^{n}(x), \operatorname{Fix}(T)\right)=0$. Let us show that $\left(T^{n}(x)\right)_{n}$ is a Cauchy sequence. Indeed, for any $n<m$ in $\mathbb{N}$, we have

$$
d\left(T^{n}(x), T^{m}(x)\right) \leq d\left(T^{n}(x), c\right)+d\left(c, T^{m}(x)\right) \leq 2 d\left(T^{n}(x), c\right),
$$

for any $c \in \operatorname{Fix}(T)$, which implies

$$
d\left(T^{n}(x), T^{m}(x)\right) \leq d\left(T^{n}(x), c\right)+d\left(c, T^{m}(x)\right) \leq 2 d\left(T^{n}(x), \operatorname{Fix}(T)\right) .
$$

Since $\lim _{n \rightarrow \infty} d\left(T^{n}(x), \operatorname{Fix}(T)\right)=0$, we conclude that $\left(T^{n}(x)\right)_{n}$ is indeed a Cauchy sequence. The completeness of $X$ will imply that $\left(T^{n}(x)\right)_{n}$ is convergent. Denote its limit by $P(x)$. It is easy to check that, since $T$ is projective, $\operatorname{Fix}(T)$ is closed. Moreover for each $n \geq 1$, there exists $c_{n} \in \operatorname{Fix}(T)$ such that

$$
d\left(T^{n}(x), c_{n}\right) \leq d\left(T^{n}(x), \operatorname{Fix}(T)\right)+\frac{1}{n} .
$$

Therefore $\left(c_{n}\right)_{n}$ also converges to $P(x)$. Since Fix $(T)$ is closed, we must have $P(x) \in$ Fix $(T)$ which implies $T \circ P=P$. The fact that $P \circ T=P$ is obvious from the properties of the iterates. The last part (ii) follows from the fact

$$
\lim _{n \rightarrow \infty} d\left(T^{n}(x), c\right)=\inf _{n \geq 0} d\left(T^{n}(x), c\right)=d(P(x), c),
$$

for any $x \in D$ and $c \in \operatorname{Fix}(T)$.

For a family of mappings, we have the following extension.

Definition 2.2. Let $(X, d)$ be a metric space and $D$ be a nonempty subset of $X$. Let $\mathcal{T}$ be a family of mappings defined from $D$ into $D$. The fixed point set of $\mathcal{T}$ is the set 
. We will say that $\mathcal{T}$ is projective w.r.t. $c \in \operatorname{Fix}(\mathcal{T})$ if any $T \in \mathcal{T}$ is projective w.r.t. c. Similarly, we will say that $\mathcal{T}$ is projective if and only if any $T \in \mathcal{T}$ is a projective mapping w.r.t. any point in $\operatorname{Fix}(\mathcal{T})$.

In the proof of the previous proposition, we used some interesting properties of the iterates. These properties are satisfied by what is known as Fejér sequences.

Definition 2.3. Let $(X, d)$ be a metric space and $D$ be a nonempty subset of $X$. A sequence $\left(x_{n}\right)_{n} \subset X$ is said to be Fejér monotone w.r.t. $D$ if

$$
d\left(x_{n+1}, c\right) \leq d\left(x_{n}, c\right) ; \text { for all } c \in D \text { and } n \geq 0 .
$$

Note that if $\left(x_{n}\right)_{n}$ is Fejér monotone then it is bounded, and $\left(d\left(x_{n}, D\right)\right)_{n}$ is monotone decreasing.

Definition 2.4. Let $(X, d)$ be a metric space. Let $\left\{C_{1}, \ldots, C_{N}\right\}$ be a finite family of nonempty subsets of $X$. We will say that $\left\{C_{1}, \ldots, C_{N}\right\}$ is boundedly regular, if for any bounded sequence $\left(x_{n}\right)_{n} \subset X$ we have

$$
\lim _{n \rightarrow \infty} \max _{i=1, \ldots, N} d\left(x_{n}, c_{i}\right)=0 \Rightarrow \lim _{n \rightarrow \infty} d\left(x_{n}, \bigcap_{i=1}^{N} C_{i}\right)=0 .
$$

We will say that $\left\{C_{1}, \ldots, C_{N}\right\}$ is innately boundedly regular if $\left(C_{i}\right)_{i \in J}$ is boundedly regular for any nonempty subset $J$ of $\{1, \ldots, N\}$.

For examples on boundedly regular sets, the reader may consult [1]. In fact this property is the one that weakens the compactness assumption used in many results.

Next, we define the concept of quasi-projection as introduced by Baillon and Bruck [19].

Definition 2.5. Let $(X, d)$ be a metric space and $C$ be a nonempty subset of $X$. Let $x_{0}$ $\in X$.

The quasi-projection of $x_{0}$ onto $C$, denoted by $\mathcal{Q}_{C}\left(x_{0}\right)$, is defined by

$$
\mathcal{Q}_{C}\left(x_{0}\right)=\left\{x \in C: d(x, c) \leq d\left(x_{0}, c\right), \text { for any } c \in C\right\} .
$$

Under the above assumptions, the definition of the projection is given by

$$
P_{C}\left(x_{0}\right)=\left\{c \in C: d\left(x_{0}, c\right)=d\left(x_{0}, C\right)\right\} .
$$

Clearly we have $P_{C}\left(x_{0}\right) \subset \mathcal{Q}_{C}\left(x_{0}\right)$. And if $x_{0} \in C$, we have

$$
P_{C}\left(x_{0}\right)=\mathcal{Q}_{C}\left(x_{0}\right)=\left\{x_{0}\right\} .
$$

\section{Main results}

Definition 3.1. Let $(X, d)$ be a metric space and $D$ be a nonempty subset of $X$. Let $\left\{T_{1}\right.$, $\left.\ldots, T_{N}\right\}$ be a finite family of mappings defined from $D$ into $D$. A mapping $T: D \rightarrow D$ is called a full word if $T$ can be written as a finite product of the mappings $\left\{T_{1}, \ldots, T_{N}\right\}$, where each $T_{i}$ will occur at least once. The set of all full word mappings will be denoted by $\mathscr{F}$. A mapping $T: D \rightarrow D$ is called a $M$-word, where $M \in\{1, \ldots, N\}$, if $T$ is a finite product of the mappings $\left\{T_{1}, \ldots, T_{N}\right\}$, where at most $M$ different $\left\{T_{i_{1}}, \ldots, T_{i_{M}}\right\}$ occur in this product for some $\left\{i_{1}, \ldots, i_{M}\right\} \subset\{1, \ldots, N\}$. The set of all $M$-words will be denoted by $\mathcal{W}_{M}$.

Note that the identity map belongs to $\mathcal{W}_{N}$, for any $M \in\{1, \ldots, N\}$, and $\mathcal{F} \subset \mathcal{W}_{N}$. 
Proposition 3.1. Let $(X, d)$ be a complete metric space. Let $\left\{T_{1}, \ldots, T_{N}\right\}$ be a finite family of mappings defined from $D$ into $D$, where $D$ is a nonempty and closed subset of $X$. Assume that $\left\{T_{1}, \ldots, T_{N}\right\}$ are projective w.r.t. a common fixed point $c \in D$. Also assume that $\left\{\operatorname{Fix}\left(T_{1}\right), \ldots, \operatorname{Fix}\left(T_{N}\right)\right\}$ is innately boundedly regular. Let $\left(x_{n}\right)_{n}$ be a bounded sequence in $D$ and $\left(W_{n}\right)_{n} \subset \mathcal{W}_{N}$ such that $\lim _{n \rightarrow \infty}\left[d\left(x_{n}, c\right)-d\left(W_{n}\left(x_{n}\right), c\right)\right]=0$. Then

$$
(*) \quad \lim _{n \rightarrow \infty} d\left(x_{n}, W_{n}\left(x_{n}\right)\right)=0 .
$$

Moreover if $\left(W_{n}\right)_{n} \subset \mathcal{F}$, then $\lim _{n \rightarrow \infty} d\left(x_{n}, \bigcap_{i=1}^{N} \operatorname{Fix}\left(T_{i}\right)\right)=0$.

Proof Consider the following statement $\mathcal{P}(M)$, where $M \in\{1, \ldots, N\}$ :

For any bounded sequence $\left(x_{n}\right)_{n} \subset D$ and any sequence of words $\left(W_{n}\right)_{n} \subset \mathcal{W}_{N}$ such that $\lim _{n \rightarrow \infty}\left[d\left(x_{n}, c\right)-d\left(W_{n}\left(x_{n}\right), c\right)\right]=0$ we must have

$$
\lim _{n \rightarrow \infty} d\left(x_{n}, W_{n}\left(x_{n}\right)\right)=0 .
$$

The proof will be done via an induction argument on $M$. First we prove $\mathcal{P}(M)$, when $M=1$. Assume it is not true. Then there exists a bounded sequence $\left(x_{n}\right)_{n} \subset D$, an $i \in$ $\{1, \ldots, N\}$ and a sequence of words $\left(W_{n}\right)_{n}$, where $W_{n}=T_{i}^{l_{n}}$ such that

$$
\lim _{n \rightarrow \infty}\left[d\left(x_{n}, c\right)-d\left(W_{n}\left(x_{n}\right), c\right)\right]=0
$$

and $\lim _{n \rightarrow \infty} d\left(x_{n}, W_{n}\left(x_{n}\right)\right) \neq 0$. Note that we have

$$
d\left(W_{n}\left(x_{n}\right), c\right)=d\left(T_{i}^{l_{n}}\left(x_{n}\right), c\right) \leq d\left(T_{i}\left(x_{n}\right), c\right) \leq d\left(x_{n}, c\right), \text { for any } n \geq 0,
$$

where the last inequality holds, since $T_{i}$ is projective w.r.t. $c$. Thus, we have $\lim _{n \rightarrow \infty}\left[d\left(x_{n}, c\right)-d\left(T_{i}\left(x_{n}\right), c\right)\right]=0$, which implies that $\lim _{n \rightarrow \infty} d\left(x_{n}, \operatorname{Fix}\left(T_{i}\right)\right)=0$. For any $z \in$ $\operatorname{Fix}\left(T_{i}\right)$, we have

$$
d\left(x_{n}, W_{n}\left(x_{n}\right)\right)=d\left(x_{n}, T_{i}^{l_{n}}\left(x_{n}\right)\right) \leq d\left(x_{n}, z\right)+d\left(T_{i}^{l_{n}}\left(x_{n}\right), z\right) \leq 2 d\left(x_{n}, z\right) .
$$

Since $z \in \operatorname{Fix}\left(T_{i}\right)$ is arbitrary, we obtain $d\left(x_{n}, W_{n}\left(x_{n}\right)\right) \leq 2 d\left(x_{n}, \operatorname{Fix}\left(T_{i}\right)\right)$. This is a contradiction. Therefore, $\mathcal{P}(M)$ is true for $M=1$. Assume $\mathcal{P}(M-1)$, for $M \in\{2, \ldots, N\}$, is true and let us prove that $\mathcal{P}(M)$ is also true. Let $\left(x_{n}\right)_{n} \subset D$ be $a$ bounded sequence and $\left(W_{n}\right)_{n} \subset \mathcal{W}_{N}$ be any sequence of words such that $\lim _{n \rightarrow \infty}\left[d\left(x_{n}, c\right)-d\left(W_{n}\left(x_{n}\right), c\right)\right]=0$. From the definition of $\mathcal{W}_{M}$, there exists $\left\{i_{1}, \ldots, i_{M}\right\} \subset\{1, \ldots, N\}$ such that each $W_{n}$ is a full word of $\left\{T_{i_{1}}, \ldots, T_{i_{M}}\right\}$ Fix $i_{m} \in\left\{i_{1}, \ldots, i_{M}\right\}$. Then we have $W_{n}=L_{n} \circ T_{i_{m}} \circ W_{n}^{*}$ where $L_{n}$ is a word generated by $\left\{T_{i_{1}}, \ldots, T_{i_{M}}\right\}$ and $W_{n}^{*}$ is a word generated by $\left\{T_{i_{1}, \ldots,}, T_{i_{M}}\right\} \backslash\left\{T_{i_{m}}\right\}$ for any $n \geq 0$. Since

$$
d\left(W_{n}\left(x_{n}\right), c\right) \leq d\left(T_{i} \circ W_{n}^{*}\left(x_{n}\right), c\right) \leq d\left(W_{n}^{*}\left(x_{n}\right), c\right) \leq d\left(x_{n}, c\right),
$$

for any $n \geq 0$, we obtain

$$
\lim _{n \rightarrow \infty}\left[d\left(x_{n}, c\right)-d\left(W_{n}^{*}\left(x_{n}\right), c\right]=0 \text { and } \lim _{n \rightarrow \infty}\left[d\left(W_{n}^{*}\left(x_{n}\right), c\right)-d\left(T_{i_{m}} \circ W_{n}^{*}\left(x_{n}\right), c\right)\right]=0 .\right.
$$

Since $\left(x_{n}\right) n$ is bounded, we deduce that $\left(W_{n}^{*}\left(x_{n}\right)\right)_{n}$ is also bounded. Using the fact that $\mathcal{P}(M-1)$ is true and $T_{i_{m}}$ is projective w.r.t. $c$, we conclude that 


$$
\lim _{n \rightarrow \infty} d\left(x_{n}, W_{n}^{*}\left(x_{n}\right)\right)=0 \text { and } \lim _{n \rightarrow \infty} d\left(W_{n}^{*}\left(x_{n}\right), \operatorname{Fix}\left(T_{i_{m}}\right)\right)=0 .
$$

This will easily imply $\lim _{n \rightarrow \infty} d\left(x_{n}, \operatorname{Fix}\left(T_{i_{m}}\right)\right)=0$. Since $i_{m}$ was arbitrarily chosen in $\left\{i_{1}\right.$, ..., $\left.i_{M}\right\}$, we conclude that

$$
\lim _{n \rightarrow \infty} \max _{i_{m} \in\left\{i_{1}, \ldots, i_{M}\right\}} d\left(x_{n}, \operatorname{Fix}\left(T_{i_{m}}\right)\right)=0 .
$$

Using the innately boundedness regularity of $\operatorname{Fix}\left(T_{i_{m}}\right)$, for $i_{m} \in\{1, \ldots, N\}$, we conclude that

$$
\lim _{n \rightarrow \infty} d\left(x_{n}, \bigcap_{i=i_{1}}^{i_{M}} \operatorname{Fix}\left(T_{i}\right)\right)=0 .
$$

Obviously, we have $\bigcap_{i=i_{1}}^{i_{M}} \operatorname{Fix}\left(T_{i}\right) \subset \operatorname{Fix}\left(W_{n}\right)$, for any $n \geq 0$. Hence we have

$$
d\left(x_{n}, W_{n}\left(x_{n}\right)\right) \leq 2 d\left(x_{n}, \bigcap_{i=i_{1}}^{i_{M}} \operatorname{Fix}\left(T_{i}\right)\right)
$$

which implies that $\lim _{n \rightarrow \infty} d\left(x_{n}, W_{n}\left(x_{n}\right)\right)=0$. This concludes that $\mathcal{P}(M)$ is true for any $M \in\{1, \ldots, N\}$. Therefore, we have proven that $\mathcal{P}(N)$ is true as well. This completes the proof of the first part of our proposition. The second part follows easily from the fact that for any $i \in\{1, \ldots, N\}$, we have $\lim _{n \rightarrow \infty} d\left(x_{n}, \operatorname{Fix}\left(T_{i}\right)\right)=0$ and the innately boundedness regularity of $\operatorname{Fix}\left(T_{i}\right)$, for $i \in\{1, \ldots, N\}$.

Note that condition (*) first appeared in Dye and Reich [20] as the condition (S).

Next, we give the main result of this article.

Theorem 3.1. Let $(X, d)$ be a complete metric space. Let $\left\{T_{1}, \ldots, T_{N}\right\}$ be a finite family of mappings defined from $D$ into $D$, where $D$ is a nonempty and closed subset of $X$. Assume that $\left\{T_{1}, \ldots, T_{N}\right\}$ are projective w.r.t. a common fixed point $c \in D$. Also Assume that $\left\{\operatorname{Fix}\left(T_{1}\right), \ldots, \operatorname{Fix}\left(T_{N}\right)\right\}$ is innately boundedly regular. Let $r: \mathbb{N} \rightarrow\{1, \ldots, N\}$ be a random mapping which assumes each value infinitely often. Then the random sequence $\left(x_{n}\right)_{n}$, defined by

$$
x_{0} \in D ; \text { and } x_{n+1}=T_{r(n)}\left(x_{n}\right), \text { for all } n \geq 0,
$$

$$
\text { converges to a point in } \mathcal{Q}_{C}\left(x_{0}\right) \text {, where } C=\bigcap_{i=1}^{N} \operatorname{Fix}\left(T_{i}\right) \text {. }
$$

Proof. Since $r$ is a random mapping, there exists a subsequence $\left(x_{n_{k}}\right)_{k}$ of $\left(x_{n}\right)_{n}$ such that $W_{k}=T_{r\left(n_{k+1}-1\right)} \circ \cdots \circ T_{r\left(n_{k}\right)}$ is a full word of $\left\{T_{1}, \ldots, T_{N}\right\}$, for any $k$. It is easy to check that $\left(x_{n}\right)_{n}$ is Fejér monotone w.r.t. C. Our assumptions and Proposition 3.1 will then imply $\lim _{n_{k} \rightarrow \infty} d\left(x_{n_{k}}, C\right)=0$. Since $\left(d\left(x_{n}, C\right)\right)_{n}$ is decreasing, we conclude that $\lim _{n \rightarrow \infty} d\left(x_{n}, C\right)=0$. As we did before, we can easily show that for any $n<m$, we have $d$ $\left(x_{n}, x_{m}\right)<2 d\left(x_{w}, C\right)$. Hence $\left(x_{n}\right)_{n}$ is a Cauchy sequence which converges to a point $\omega \in$ $D$. Since $\left(x_{n}\right)_{n}$ is Fejér monotone w.r.t. $C$, we have 


$$
d(\omega, c)=\lim _{n \rightarrow \infty} d\left(x_{n}, c\right)=\inf _{n \geq 0} d\left(x_{n}, c\right)
$$

for any $c \in C$. In particular, we have $d(\omega, c) \leq d\left(x_{0}, c\right)$ for any $c \in C$. Moreover since $C$ is closed and $\lim _{n \rightarrow \infty} d\left(x_{n}, C\right)=0$, we conclude that $\omega \in C$. Putting everything together we obtain that $\omega \in \mathcal{Q}_{C}\left(x_{0}\right)$. This proves Theorem 3.1.

\section{Acknowledgements}

The authors gratefully acknowledge the financial support from King Fahd University of Petroleum \& Minerals for supporting research project SB 101021. The authors would also like to thank the referees for excellent suggestions and useful comments.

\section{Author details}

${ }^{1}$ Department of Mathematical Sciences, The University of Texas At El Paso, El Paso, TX 79968, USA ${ }^{2}$ Department of Mathematics and Statistics, King Fahd University of Petroleum \& Minerals, Dhahran 31261, Saudi Arabia

\section{Authors' contributions}

All authors participated in the design of this work and performed equally. All authors read and approved the final manuscript.

\section{Competing interests}

The authors declare that they have no competing interests.

Received: 28 September 2011 Accepted: 20 March 2012 Published: 20 March 2012

\section{References}

1. Bauschke, HH: A norm convergence result on random products of relaxed projections in hilbert space. Trans Am Math Soc. 347(4):1365-1373 (1995)

2. Deutsch, F, Singh, SP: The method of alternating orthogonal projections. Approximation Theory, Spline Features and Applications. pp. 105-121.Kluwer Academic Amsterdam (1992). Proc Conf Hotel Villa del Mare Maratea Italy April 28 1991 May 91991

3. Censor, Y: Parallel application of block-iterative methods in medical imaging and radiation therapy. Math Program. 42 , 307-325 (1988)

4. Censor, Y, Herman, GT: On some optimization techniques in image reconstruction from projections. Appl Numer Math. 3, 365-391 (1987)

5. Sezan, Ml: An overview of convex projections theory and its applications to image recovery problems. Ultramicroscopy. 40, 55-67 (1992)

6. Bauschke, HH, Borwein, JM: On projection algorithms for solving convex feasibility problems. Siam Rev. 38(3):367-426 (1996)

7. Combettes, PL: Hilbertian convex feasibility problem: convergence of projection methods. Appl Math Optim. 35 , 311-330 (1997)

8. Amemiya, I, Ando, T: Convergence of random products of contractions in Hilbert space. Acta Sci Math (Szeged). 26, 239-244 (1965)

9. Bruck, RE: Random products of contractions in metric and Banach spaces. J Math Anal Appl. 88, 319-332 (1982)

10. Dye, JM: Convergence of random products of compact contractions in Hilbert space. Integral Equ Oper Theory. 12, 12-22 (1989)

11. Dye, JM, Reich, S: Unrestricted iterations of nonexpansive mappings in Hilbert space. Nonlinear Anal. 18, 199-207 (1992)

12. Youla, DC: On deterministic convergence of iterations of relaxed projection operators. J Visual Com-mun Image Represent. 1, 12-20 (1990)

13. Aharoni, R, Censor, Y: Block-iterative projection methods for parallel computation of solutions to convex feasibility problems. Linear Algebra Appl. 120, 165-175 (1989)

14. Flam, SD, Zowe, J: Relaxed outer projections, weighted averages and convex feasibility. BIT. 30, 289-300 (1990)

15. Tseng, P: On the convergence of the products of firmly nonexpansive mappings. SIAM J Optim. 2, $425-434$ (1992)

16. Eisner, L, Koltracht, I, Neumann, M: Convergence of sequential and asynchronous nonlinear para-contractions. Numer Math. 62, 305-319 (1992)

17. Pustylnik, E, Reich, S, Zaslavski, AJ: Convergence of non-cyclic infinite products of operators. J Math Anal Appl. 380, 759-767 (2011)

18. Dye, JM, Khamsi, MA, Reich, S: Random products of contractions in Banach spaces. Trans Am Math Soc. 325(1):87-99 (1991)

19. Baillon, JB, Bruck, RE, Tan, KT: Ergodic theorems and the asymptotic behavior of contraction semigroups. Fixed Point Theory and Applications. pp. 12-26.World Scientific Publ., Singapore (1992). Proc. Internat. Conf., Halifax, Nova Scotia, Canada, June 9-14, 1991

20. Dye, JM, Reich, S, lofFe, A, Marcus, M, Reich, S: Random products of nonexpansive mappings. In Optimization and Nonlinear Analysis, vol. 244, pp. 106-118.Longman Sci. Tech, Harlow, England (1992). Proc. Binational Workshop on Optimization and Nonlinear Analysis, Technion City, Haifa, $21-27$ March 1990, Pitman Res. Notes in Math. Ser. 